\title{
An attempt to find changes of post-translational modifications on serum proteins between embryonic I5.5 fetuses and newborn rats
}

\begin{abstract}
Post-translational modifications (PTMs) relate to protein functions via changing of their physicochemical properties. In order to better understand and evaluate the function of plasma, changes in serum protein PTMs between embryonic 15.5 (E 15.5) fetuses and newborn rats were investigated using high throughput proteomics techniques and an unrestrictive search strategy on PTM analysis. In this study, triplicate analyses of liquid chromatography coupled with tandem mass spectrometry (LC-MS/MS) were performed on the pooled specimens of E15.5 fetuses and newborn rats respectively, and single analyses of LC-MS/MS were performed on three individual specimens. The PEAKS 6 software was used for PTMs analysis and some PTMs were identified using the search parameters set in the software. In this preliminary study, most PTM ratios were extremely low and the modified peptide number was very small. This is the first attempt to explore the dynamic change of plasma PTMs during rat development.
\end{abstract}

Keywords: serum proteome, post-translational modification (PTM), nonenrichment, rat serum, LC-MS/MS, serum development
Volume 6 Issue I - 2017

\author{
Lilong Wei,' Zhigang Wang, ${ }^{3}$ Lisi Zhu, ${ }^{3}$ Youhe \\ $\mathrm{Gao}^{2}$ \\ 'Department of Laboratory Medicine, China-Japan Friendship \\ Hospital, China \\ ${ }^{2}$ College of Life Sciences, Beijing Normal University, China \\ ${ }^{3}$ Department of Physiology and Pathophysiology, National Key \\ Laboratory of Medical Molecular Biology, China
}

Correspondence: Youhe Gao, College of Life Sciences, Beijing Normal University, Beijing, China 100875, Fax 861001058807721,Email gaoyouhe@bnu.edu.cn I

Received: January 22, 2017| Published: August 03, 2017
Abbreviations: PTMs, post-translational modifications; LC, liquid chromatography; MS, mass spectrometry

\section{Introduction}

Blood is one of the most complex body fluids and plays important functions such as metabolic nutrient transport, signal conduction and immuno-modulatory. Proteins, the most important component of plasma, are considered the main executors of the physiological function of blood. ${ }^{1}$ Post-translational modifications (PTMs) of proteins can regulate protein functions by changing the physicochemical properties. ${ }^{2}$ For example, phosphorylation or dephosphorylation may turn the kinase cascades in signaling on or off, ${ }^{3}$ and is often associated with tumorigenesis. So far, more than 500 types of PTMs or biological and chemical modifications have been identified and documented in the PSI-MOD ${ }^{4}{ }_{\text {RESID }}{ }^{5}$ UniProt $^{6}$ and UniMod ${ }^{7}$ databases. These translational modifications may determine the diversity of protein functions. ${ }^{8}$ Research on post-translational modifications of plasma proteins with different physiological states could help to better understand and evaluate the function of plasma.

Identification of post-translational modification plays a key role in gaining insight into the biological functions of protein but remains a challenge before proteomic era. Tandem mass spectrometry (MS/ MS) could be a powerful tool for rapid identification of PTMs by analyzing PTM-related diagnostic mass shifts of fragment ions in MS/ MS spectra. ${ }^{9,10}$ Principally, qualitative and quantitative information of protein PTMs on a global scale could be obtained based on mass spectrometry. However, currently, most studies on PTMs have been based on the enrichment of modified proteins or peptides and twodimensional gel electrophoresis. ${ }^{11}$ This has been hampered by a lack of affinity methods for the nearly one thousand different types of modifications. In recent years, with the increased accuracy and resolution of the latest generation of mass spectrometry (MS) ${ }^{12}$ as well as several developed search algorithms for PTM detection, ${ }^{13}$ the interest in large-scale analyses of PTMs has rapidly increased. ${ }^{14}$ Some studies based on unrestrictive search strategies were applied to PTM identification. ${ }^{15-17}$

Plasma proteins changed dramatically with the development of serum proteomes of newborn rats and E15.5 fetuses. ${ }^{18}$ However, there have been only a few research projects focusing on protein modifications of plasma. For example, protein carbonyls in the serum of fetal and neonatal pigs were identified by 2D gel electrophoresis using biotin labeling. ${ }^{19}$

This study is the first attempt to analyze PTMs changes of serum protein between E15.5 fetuses and newborn rats using unrestrictive search strategy of MS-based PTM identifications.

\section{Materials and methods}

\section{Experiment design}

An unrestrictive search strategy was used to study the PTM of E15.5 fetus and newborn rat serum proteins. In this study, both pooled and individual specimens were analyzed by liquid chromatography coupled with tandem mass spectrometry (LC-MS/MS). Then, identification of PTMs was performed using the PEAKS 6 software.

\section{Sample preparation}

The study was authorized by the Institute of Basic Medical Sciences Animal Ethics Committee at the Peking Union Medical College. Animals were caged and handled according to international rules of animal care specified in the International Animal Welfare Recommendations. Sprague-Dawley rats were purchased from the Huafukang Biotechnical Company (Beijing, China). Specimens of E15.5 fetuses and newborn rats were obtained and processed as previously described. ${ }^{20}$ 


\section{Mass Spectrometry (MS) analysis}

In this study, triplicate analyses of LC-MS/MS were performed on the pooled specimens for E15.5 fetuses and newborn rats respectively. Single analyses of LC-MS/MS were performed on individual specimens. Proteins were digested using a previously used method. ${ }^{21}$ The tryptic peptides were analyzed using LC-MS/MS. The peptides were sequentially loaded onto a Michrom peptide Captrap (MW 0.5$50 \mathrm{kD}, 0.5 \times 2 \mathrm{~mm}$; Michrom Bioresources, Inc, FOB Auburn, CA, USA) at a flow rate of $20 \mu \mathrm{L} / \mathrm{min}$ with a mobile phase $(0.1 \%$ formic acid, $99.9 \%$ water). They were then transferred to a reversed-phase micro capillary column $(0.1 \times 150 \mathrm{~mm}$, packed with Magic C18, $5 \mu \mathrm{m}$, 200Å; Michrom Bioresources, Inc, FOB Auburn, CA, USA) in an Eksigent system (AB, Inc, Framingham, Massachusetts, USA). Separation of the peptides was performed at a flow rate of $500 \mathrm{~nL} / \mathrm{min}$ and coupled to an online analysis by tandem MS using LTQ Orbitrap Velos (Thermo Fisher Scientific, San Jose, USA). The elution gradient for the reverse column and the MS program were detailed in previous studies. ${ }^{18}$

\section{PTM identification}

The files were processed using the PEAKS 6 software and the analysis parameters were set as follows: Parent Mass Error Tolerance: 10.0ppm, Fragment Mass Error Tolerance: 0.1Da, Precursor Mass Search Type: Monoisotopic, Max Missed Cleavages: 2, Non-specific Cleavage: 1, Fixed Modifications: Carbamidomethylation: 57.02, Variable Modifications: Deamidation (NQ): 0.98; Oxidation (M): 15.99; Pyroglu from Q: -17.03; 4-hydroxynonenal (HNE): 156.12; Acetylation(K): 42.01; Acetylation(N-term): 42.01; Acetylation (Protein N-term): 42.01; Amidation: -0.98; and 669 more built-in modifications in PEAKS 6; Max variable PTM per peptide: 3; Result filtration parameters: De novo score (ALC\%) threshold: 30; Peptide $-10 \lg \mathrm{P} \geq 30$; Protein $-10 \operatorname{lgP} \geq 60$; FDR (Peptide-Spectrum Matches): $1.00 \%$; Database: ipi.RAT.v3.78.fasta.

Following the data search, the observed modification name was matched with the modification classification on the Unimod website. Because some kinds of modifications are noted as Artifact, Chemical derivative, AA substitution, Pre-translational, Multiple as well as Post-translational, the PTMs that are classified as 'Post-translational' were analyzed. ${ }^{22-24}$

\section{Results and discussion}

In this work, triplicate analyses of LC-MS/MS were performed on the pooled specimens of E15.5 fetuses and newborn rats respectively. Single analyses of LC-MS/MS were performed on individual specimens. All specimens were analyzed by tandem MS using LTQ Orbitrap Velos and the data were searched using PEAKS software with FDR (Peptide-Spectrum Matches): 1.00\%. Identified modifications are listed in Table 1 . In this preliminary study, most PTM ratios were extremely low and the modified peptide number was very small (Table $2 \& 3$ ).

There are many software packages for searching known and unknown PTMs. However, PTM analysis using MS still has a number of challenges ${ }^{25}$ to surmount the most common errors that can occur during MS-based analysis of PTMs and lead to incorrect data interpretation and erroneous conclusions. ${ }^{26,27}$ More reliable PTMs may be identified based on the enrichment of modified proteins or peptides in the future. With the development of mass spectrometry and software analysis techniques, blood post-translational modifications at different stages of development deserve further research.
This study is the first attempt to explore the dynamic change of plasma PTMs during rat development.

\section{Acknowledgements}

National Key Research and Development Program of China (2016YFC 1306300), National Basic Research Program of China (2013CB530805), Beijing Natural Science Foundation (7173264,7172076), the Fundamental Research Funds for the Central Universities (2015KJJCB21) Beijing cooperative construction project (110651103) Beijing Normal University(11100704), China-Japan Friendship Hospital research fund (2016-1-QN-5).

\section{Author contribution}

Lilong Wei and Youhe Gao were responsible for planning and designing the study. Lilong Wei collected the samples and performed the experiments. Lisi Zhu performed the PTM identification. Zhigang Wang and Lilong Wei performed the data analysis. Lilong Wei wrote the manuscript. Youhe Gao revised the manuscript. All authors read and approved the final manuscript.

\section{Conflict of interest}

The author declares no conflict of interest.

\section{References}

1. Anderson NL, Anderson NG. The human plasma proteome: history, character, and diagnostic prospects. Mol Cell Proteomics. 20021(11):845867.

2. ON Jensen. Interpreting the protein language using proteomics. Nature Reviews Molecular Cell Biology. 2006;7(6):391-403.

3. Cohen P. The regulation of protein function by multisite phosphorylation-a 25 year update. Trends Biochem Sci. 2000;25 (12):596-601.

4. Montecchi-Palazzi L, Beavis R, Binz PA, et al. The PSI-MOD community standard for representation of protein modification data. Nat Biotechnol. 2008;26(8):864-866.

5. Garavelli JS. The RESID Database of Protein Modifications as a resource and annotation tool. Proteomics. 2004;4(6):1527-1533.

6. UniProt Consortium. UniProt: a hub for protein information. Nucleic Acids Res. 2015;43(Database issue):D204-D212.

7. Creasy DM, Cottrell JS. Unimod: Protein modifications for mass spectrometry. Proteomics. 2004;4(6):1534-1536.

8. Muñoz J, Heck AJ. From the human genome to the human proteome. Angew Chem Int Ed Engl. 2014;53(41):10864-10866.

9. Lu B, McClatchy DB, Kim JY, et al. Strategies for shotgun identification of post-translational modifications by mass spectrometry. J Chromatogr A. 2004;8(19):3947-3955.

10. Steen H, Mann M. The ABC's (and XYZ's) of peptide sequencing. Nat Rev Mol Cell Biol. 2004;5(9):699-711.

11. Mann M, Jensen ON. Proteomic analysis of post-translational modifications. Nat Biotechnol. 2003;21(3):255-261.

12. Mann M, Kelleher NL. Precision proteomics: the case for high resolution and high mass accuracy. Proc Natl Acad Sci USA. 2008;105(47):1813218138 .

13. Zybailov B, Sun Q, van Wijk KJ. Workflow for large scale detection and validation of peptide modifications by RPLC-LTQ-Orbitrap: application to the Arabidopsis thaliana leaf proteome and an online modified peptide library. Anal Chem. 2009;81(19):8015-8024. 
14. M Larsen MR, Trelle MB, Thingholm TE, et al. Analysis of posttranslational modifications of proteins by tandem mass spectrometry. Biotechniques. 2006;40(6):790-798.

15. Na S, Bandeira N, Paek E. Fast multi-blind modification search through tandem mass spectrometry. Mol Cell Proteomics. 2012;11(4):M111.010199.

16. V Schwämmle V, Verano-Braga T, Roepstorff P. Computational and statistical methods for high-throughput analysis of post-translational modifications of proteins. J Proteomics. 2015;129:3-15.

17. Liu L, Liu X, Sun W, et al. Unrestrictive identification of posttranslational modifications in the urine proteome without enrichment Proteome Sci. 2013;11(1):1.

18. Wei L, Jia L, Zhu L, et al. A comparison of E15.5 fetus and newborn rat serum proteomes. Proteome Sci. 2012;10(1):64.

19. Caperna TJ, Shannon AE, Blomberg Le Ann, et al. Identification of protein carbonyls in serum of the fetal and neonatal pig. Comp Biochem Physiol B Biochem Mol Biol. 2010;156(3):189-196.

20. Heim WG. The Serum Proteins of the Rat During Development. $J$ Embryol Exp Morphol. 1961;52:59.

21. Sun W, Li F, Wu S, et al. Human urine proteome analysis by three separation approaches. Proteomics. 2005;5(18):4994-5001.
22. Shortreed MR, Wenger C1, Frey B1, et al. Global Identification of Protein Post-translational Modifications in a Single-Pass Database Search. J Proteome Res. 2015;14(11):4714-4720.

23. Chen Y, Chen W, Cobb MH, et al. PTMap - a sequence alignmen software for unrestricted, accurate, and full-spectrum identification of post-translational modification sites. Proc Natl Acad Sci U S A. 2009;106(3):761-766.

24. Kertész-Farkas A1, Reiz B, Vera R, et al. PTMTreeSearch: a nove two-stage tree-search algorithm with pruning rules for the identification of post-translational modification of proteins in MS/MS spectra. Bioinformatics. 2014;30(2):234-241.

25. Kim M, Zhong J, Pandey. A Common errors in mass spectrometrybased analysis of post-translational modifications. Proteomics. 2015;16(5):700-714

26. Palmisano G, Melo-Braga MN, Engholm-Keller K, et al. Chemica deamidation: a common pitfall in large-scale N-linked glycoproteomic mass spectrometry-based analyses. J Proteome Res. 2012;11(3):19491957.

27. Nielsen ML, Vermeulen M, Bonaldi T, et al. Iodoacetamide-induced artifact mimics ubiquitination in mass spectrometry. Nat Methods. 2008;5(6):459-460. 